\title{
Illness Remission: Anathema to Compliance with Hypertensive Therapy
}

\author{
Joel Olayiwola Faronbi ${ }^{1,}$, , Ayodele Feyi Ojo ${ }^{1}$, Alinane Linda Nyondo Mipando ${ }^{2}$ \\ ${ }^{1}$ Department of Nursing Science, College of Health Science, Obafemi Awolowo University, Ile-Ife, Nigeria \\ ${ }^{2}$ Department of Health Systems, School of Public Health and Family Medicine, College of Medicine, University of Malawi, Blantyre, \\ Malawi
}

Email address:

jfaronbi@oauife.edu.ng (J. O. Faronbi), faronbiy2k@yahoo.co.uk (J. O. Faronbi)

${ }^{*}$ Corresponding author

\section{To cite this article:}

Joel Olayiwola Faronbi, Ayodele Feyi Ojo, Alinane Linda Nyondo Mipando. Illness Remission: Anathema to Compliance with Hypertensive Therapy. Central African Journal of Public Health. Vol. 4, No. 3, 2018, pp. 69-75. doi: 10.11648/j.cajph.20180403.13

Received: June 2, 2018; Accepted: June 28, 2018; Published: August 4, 2018

\begin{abstract}
Background: Hypertension is a chronic and persisting illness that can be difficult to treat. The success of management of hypertension depends on adherence to the treatment regimen. The objective of this study was to explore barriers and facilitators to compliance with hypertensive drug treatment among patients in Nigeria. Method: Twenty hypertensive patients were purposively selected and participated in in-depth interviews where their experiences with hypertensive treatments were explored. Interview sessions were audio recorded and transcribed verbatim and imported into Nvivo. Data was analysed using content analysis. Results: Four interrelated themes explain barriers to full compliance among to treatment among hypertensive patients as follows: (i) Temporary remission of symptoms, (ii) Dissatisfaction with Service, (iii) Navigating the Health Care Services, and (iv) Desire for Improved Functionality. Conclusion: This study concluded that lack of understanding of the chronical nature of hypertension hinders the participants from complying with treatment. It also identified inaccessible and poor quality services as a barrier to seeking treatment while the desire to regain functionality serves as a significant impetus for continuing with the treatment. Therefore, health professionals should endeavour to provide adequate health education on compliance with treatment while government should provide quality and affordable hypertensive care to citizens.
\end{abstract}

Keywords: Barrier, Compliance, Hypertensive, In-Depth Interview, Motivator and Remission

\section{Introduction}

Hypertension is one of the most prevalent chronic noncommunicable diseases (NCDs) worldwide, both in developed and developing countries [1]. It is associated with 45 per cent of deaths due to heart disease and 51 per cent of deaths due to stroke [2]. The global prevalence trend of hypertension continues to increase [3]. According to WHO, Africa has the highest prevalence of hypertension globally with 46 per cent of adults aged 25 and above affected [2, 4]. Hypertension affects about 56 million Nigerians, accounting for 30 per cent of the population and is also common among men and women [5]. A meta-analysis result estimated the countrywide prevalence to be between 12.4 per cent and 34.8 per cent [6]. Ogundipe and Obinna [7] opined that from this revelation of the unacceptably overwhelming prevalence of hypertension within the Nigerian populace, it is expected that there should be a prompt and effective response to curb the disease.

Although hypertension is a lifelong condition, studies have demonstrated that the treatment of mild-to-moderate hypertension can reduce the risk of stroke by 30 to 43 per cent [8]. The difficulty with the treatment of hypertension is often associated with non-compliance. Patient non-adherence can take many forms ranging from misunderstood information, incorrectly carried out medication instruction, forgotten, or even wholly ignored information [9].

Poor medication adherence is an important problem in the management of hypertension [10]. The impact of noncompliance greatly affects the treatment outcomes and also direct clinical consequences [11]. One of these consequences 
includes poorly controlled blood pressure, thus increasing the risk of stroke, myocardial infarction, and renal impairment markedly [11]. Non-compliance has also been associated with patients withdrawing from treatments which limit the benefits a patient would achieve from the treatment effects [12]. In addition, the undesirable impact on clinical outcomes, non-compliance would also cause an increased financial burden on society [12] and [13]. Furthermore, low rates of adherence to therapies for asthma, diabetes, and hypertension contribute substantially to the human and economic burden of those conditions [11].

Several efforts have been made to study factors associated with non-compliance among patients with chronic illnesses Amira and Okubadejo [14], [15] and [16]. Most of the efforts were released through quantitative approaches without exploring the patient's lived experiences in the management of hypertension. The formulation of effective and acceptable interventions in addressing the problem associated with noncompliance requires qualitatively examined factors among hypertensive patients to gain depth on the problem. Having a qualitative understanding of these factors will add to the quest of identifying factors that may be used in making appropriate recommendations to the clients, the healthcare practitioners and policy-makers. Therefore, the objective of the study was to identify factors associated with compliance to treatment among patients living with hypertension attending care in Obafemi Awolowo University Teaching Hospitals Complex (OAUTHC, Ile-Ife, Nigeria.

Theoretical Framework: The Health Belief Model

The Health Belief Model [17] served as the theoretical framework for this study. The basic concepts of the model are perceived susceptibility, perceived severity, perceived benefits and perceived barriers. The model prescribes that the likelihood of patients complying with their treatment depends on the degree to which they perceive themselves to be susceptible to the disease or its complications. It also includes their perceptions of the severity of the condition and to which they believe the disease is capable of affecting/hurting their quality of life [17]. This perceived threat of the disease and effect on wellbeing are weighed against the benefits to be derived from compliance with the prescribed treatment and the barriers they need to overcome. The Health Belief Model postulates that a high perceived threat; low perceived barriers and a high-perceived benefit would result in the likelihood of exhibiting compliance behaviour.

\section{Methods}

This study was conducted at the Cardiology Clinic of Obafemi Awolowo University Teaching Hospitals Complex, Ile-Ife, Nigeria. The hospital provides general primary, secondary and tertiary level care, including cardiology for clients within and outside Osun State. The hospital is a referral centre for most hospitals in Osun state and the neighbouring states. Patients discharged from the wards are followed-up at the Consultant Outpatient Department
(COPD). Hypertensive patients are attended to at the Consultant's Clinic which takes place every Thursday with an average of 60 patients attending the clinic every week. Patients above 18 years of age who have been diagnosed with and receiving treatment for hypertension for at least minimum of three months at the Cardiology Unit of OAUTHC were included in the study. It employed a qualitative descriptive study using in-depth interviews to explore the experience of hypertensive patients in compliance with therapy was. Participants were approached during the clinic session and appointments were made for the appropriate place and time for the interview sessions. Some participants were interviewed in a quiet location within the hospital while we scheduled appointments with others at a convenient place for the interview session.

\subsection{Sample Size}

The in-depth interviews involved twenty purposively selected participants comprising nine males and eleven females.

\subsection{Data Collection}

Data was collected using an interview guide consisting of open-ended questions that explored challenges associated with compliance with hypertensive therapy. The guide further examined factors that motivate respondents to adhere to treatment. The authors also reviewed it and we, all agreed that it is suitable for the study. A registered nurse conducted the interview, and each session lasted for an average of 45 minutes and was audio-recorded and transcribed verbatim.

\subsection{Data Management and Analysis}

At the end of the session, participants were met and responses verified and checked for accuracy before data analysis. The directed content analysis was employed for the analysis. This involves starting with a theory or relevant research findings to guide the selection of codes, which is followed by an in-depth round of coding where additional themes are captured from the selected text [18]. The Health Belief Model guided the transcript coding. The transcript text was imported into NVivo (QSR N10), qualitative data analysis and management software package.

Emergent codes that appeared in the course of reading the transcript were also included. The transcript was read and reread, and all words and phrases that captured the experience of respondents with the treatment of hypertension were captured. The report was compiled and read at least three times and organised into key themes. Similar themes were grouped and emerged as major themes. To ensure trustworthiness of the data, transcripts were read at least three times, and results of the coding system were compared.

\subsection{Validity and Reliability}

To ensure validity (or 'trustworthiness') of the findings of the qualitative data, researchers prolonged engagement and member checking were employed as recommended by 
Strauss \& Corbin [19]. Researcher's prolonged engagements ensure constant interaction with the data until data saturation was reached. Similarly, member checking was also done by visiting two of the participants to confirm if they agree with the preliminary findings of the study. Also, the study got approval from appropriate institutions.

\subsection{Ethical Consideration}

Ethical approval for the study was obtained from the Ethics and Research Committee, OAUTHC, Ile-Ife. Permission was also obtained from the authority of the institution, before the commencement of the study. Participants were all volunteers, and guaranteed anonymity and confidentiality of the information provided.

\section{Results}

\section{Characteristics of respondents}

Fifty per cent of the participants were between $60-69$ years of age, $55 \%$ were female, and $46.7 \%$ were selfemployed, and most (90\%) of them were Christian. Similarly, half $(50 \%)$ of the participants were retirees, and a majority $(40 \%)$ were from Ile-Ife (Table 1)

Table 1. Socio-demographic-economic Characteristics of Respondents $n=20$.

\begin{tabular}{lll}
\hline Demographic Characteristics & Frequency & Percentage \\
\hline Age & & \\
$40-49$ & 4 & 20 \\
$50-59$ & 4 & 20 \\
$60-69$ & 10 & 50 \\
$>=70$ & 2 & 10 \\
Religion & & \\
Christian & 18 & 90 \\
Jehovah witness & 1 & 5 \\
Islam & 1 & 5 \\
Sex & & \\
Female & 11 & 55 \\
Location & & \\
Ile-Ife & 9 & 45 \\
Modakeke & 3 & 15 \\
Ilesha & 3 & 15 \\
Osogbo & 1 & 5 \\
Garage Olode & 1 & 5 \\
Ondo State & 3 & 15 \\
Occupation & & \\
Business & 3 & 15 \\
Civil servant & 3 & 50 \\
Artisan & 3 & 5 \\
Retired & 10 & \\
Teacher & 1 & \\
\hline & & \\
\hline
\end{tabular}

The following major interrelated themes were uncovered from the analysis of data using content analysis (i) Temporary remission of symptoms (ii) Dissatisfaction with Service (iii) Navigating the Health Care Services, and (iv) Desire for Improved Function

(i) Temporary Remission of Symptoms

Chronic illness is often associated with a period of remission characterised by the absence of symptoms. This period of remission may mimic a temporary cure whereby patients feel that they are well and do not need medical assistance. This false assurance affects their compliance with the therapeutic regimen. Some patients have a wrong perception that absence of symptoms signifies a complete cure, thereby putting away their drugs and other therapeutic regimens. Failure of the health care providers to adequately educate patients may even lead to a more significant challenge. Some of them claimed that once they did not have any symptom of illness, they felt that they were completely healed. This false impression made most of the respondents not only to do away with their drugs but also to abandon clinic appointment which should have afforded them the opportunity to check their blood pressure regularly and listen to medical advice.

It was not easy taking the drug every day, and when I did not feel any headache again, I stop the drug... (41 years old male civil servant/2years diagnosed).

Why should I take my drug since I am okay (55 years old man).

I stopped taking my drug or go to the clinic when I felt better, but when the sickness starts all over again, I have to start using it (45years old male teacher/2years diagnosed).

(ii) Dissatisfaction with Service

The quality of service can be defined as a comprehensive look at all aspects of a patient's experience. Quality of service can be measured in other ways, such as the amount of time spent with each patient, making sure appointments are on time, ensuring all test results and other patient information are available to every doctor whenever they are needed.

Some identified healthcare facility and service-related factors including unpleasant experience during the clinic, weak or inadequate drug information, the ambiguity of prescription and poly-pharmacy induced boredom. Public servants claimed that they found it difficult meeting up the clinic's appointment. They complained that they found it difficult waiting all through the day to see the doctor on the clinic day. They also reiterated that because of their official engagement, they could not afford the luxury of time to sit down and be waiting to see the doctor. People with hypertension need some time out from their work or studies to attend the clinic, and many complained of challenges of living up to the expectation at work and at the same time keeping up the clinic appointments. Some of our respondents reported they have to miss work or school to meet up with doctors' appointment. They also mentioned that some patients have to abandon the clinic visit because of their jobs. One of the interviewees gave this expression:

They waste our time a lot in this hospital; it is not suitable for civil servant ... (45years old female/6years diagnosed).

I come every month. But in August I was admitted here after missing my clinic appointments and stressed out. I had a programme at work which took me to Abuja; I couldn't come to the clinic on time because I have to be in Abuja... (42years old female civil servant/8years diagnosed).

Poor understanding of the direction of the treatment is a major barrier to completing treatments. Some participants narrated their experience as follows: 
The drugs are too many; the instructions are too many also, I don't know which one to follow. The information given to treatment and drugs are not enough and even confusing (81years old female/12years diagnosed).

They gave me too many drugs and I stopped using it because it is not easy eating drug-like food every day. (45years old male teacher/2years diagnosed).

I don't know which of drugs to take. They are too many; it is as if one is eating food. I don't know, how long will I have to depend on this? (70years old female/10years diagnosed).

(iii) Navigating the Health Care Services

Accessing health services is a challenge. Most of the respondents expressed their difficulty in accessing health care services in this setting. Some factors identified include unavoidable treatment cost, distance, and transportation. Patients identified financial difficulty as one of the major factors influencing compliance with treatment or clinic appointment. Cost of managing chronic illness such as hypertension is enormous and may be beyond the reach of an average person. Some patients found it difficult getting money to pay for their transportation as well as money for purchasing drugs.

For the short period that I came here for treatment in the past, they did well, but the only thing that I notice is the financial burden. I could remember a day I came with just One thousand Naira $(1,000, \approx \$ 50)$ out of which I will pay for transportation and at the same time buy drugs (61 years old retired male teacher/5years diagnosed).

Most of the respondents were retirees, and the only source of finance they have is a pension that is not regular. Out of frustration, a pensioner has this to say:

The only concern I have is that the State Government refuse to pay our pension out of which I can buy drugs (a 60 years old retiree/5years diagnosed).

A similar response from another man who claimed that

Sometimes, I bring my car, but today I didn't have money for fuel so come by public transportation. Now I don't know how I am going to get my drug (41 years old male teacher/2years diagnosed).

Respondents whose houses were not far away from the hospital trek while others who live in a faraway place had to convey themselves either by car or motorcycle. A sixty-yearold retired married man recounted his ordeal in accessing health care service:

I usually come from a long distance, Garage Olode, before I arrive most people would have submitted their card, and you know it is on the first come first serve. If you came too late, you might be turned back and asked to return on another clinic day (60years old male farmer from Garage Olode/7years diagnosed).

(iv) Desire for Improved Function

Majority of participants stated that the desire to recover from the illness is a motivation for their participation and compliance with treatment regimen. The desire for an improved health condition and optimal functional health prompted many to comply with the therapeutic regimen. Effective management of hypertension is hinged strongly on compliance with treatment regimen. The statements below illustrate why patients adhere or defaulted in taking their drugs as prescribed. Some respondents highlighted that:

I take my drug regularly so that I can be healthy, you know if you use it well you will be healthy (40years old woman civil servant/3 month diagnosed).

I have to abide by all the treatment instruction because I want to be free from all the symptoms, I was told that if I take my drug regularly, all the symptoms will go (40years old business man/lyear diagnosed).

I know that if I don't comply with it, I am only doing myself harm. So I have to comply with it, to make my health perfect ... (50years old male civil/2years diagnosed).

\section{Discussion}

This study described the motivations and barriers associated with compliance with treatment among hypertensive patients attending care at Obafemi Awolowo University Teaching Hospitals Complex, Ile-Ife, Nigeria. The barriers to compliance were secondary to a false perception of complete cure as a result of the absence of symptoms and the perceived failure of the health system while the motivating factors were their willingness to get well and be independent of the health professionals. This study identified that majority of the participants complied poorly with the treatment regimen. One of the major obstacles to the effective delivery of healthcare globally is non-compliance [20]. Estimate suggests that only about $50 \%$ of patients with chronic diseases follow treatment recommendations [21]. Low compliance with prescribed medical interventions remains a complex problem, especially for patients with chronic illness symptoms [11]. Furthermore, low compliance has been identified as a major public health problem that imposes a considerable financial burden upon modern health care systems [22]. This burden has been estimated to cost $\$ 100$ billion each year in the USA [11]. van Boven, Chavannes [11] further identified lack of compliance with medical advice is also a source of ongoing frustration to doctors and other healthcare providers.

Our results on barriers to compliance among hypertensive clients resonate with findings from a study by de PascualVerdú, Rocha-Díaz [23], Brown and Bussell [24] which showed that secondary effects of the treatment itself were associated with lower compliance with and abandonment of medication treatment. Furthermore, non-adherence to antihypertensive medication was related to the attitudes of the patients and included forgetfulness, perceived side effects of the medicines such as dehydration, loss of interest in sex, easily fatigued, palpitations, feeling hot and sweating [25]. The risk factors for poor adherence are poor socioeconomic status, illiteracy and unemployment [25] understanding and acceptance of the disease, as well as perception of the health risk related to the disease [26]. Consequently, poor adherence to anti-hypertensive treatment leads to sub-optimal bloodpressure control [10].

Compliance is determined by the knowledge and attitudes 
of the patient [27]. Patients must believe that they are vulnerable or susceptible to the disease or its consequences, that they have it, and that the consequences of the disease on their well-being could be serious [27]. They must believe that by following a particular set of health recommendations, the threat or severity of the condition will be abolished or reduced [22].

An important finding of the current study is that the desire of the participants to have an improved condition is a major motivation in complying with treatment. This is similar to the findings from Jurkiewicz, Marzolini [28] who reported the importance of patient's interest in their study among stroke patients. Findings from their study revealed that compliance with treatment is hampered by poor understanding of the course of chronic illness such as hypertension. Respondents have a wrong perception thinking that a temporary absence of illness implies that the illness is completeness and there is no need to continue taking the drug.

The compliance of patients to drug regime hinged on the fact that they wanted to be healthy and subsequently weaned off from the list of the healthcare staff. Similarly, a study by Sabaté [29] which stated that compliance of patients is strongly influenced by the patient-perceived benefit and experience of the physician as patients of older physicians were less complying unlike those seen by younger health care providers.

Compliance to clinic appointment was borne out of fear of the deteriorating health. Meanwhile, non-compliance to drug regimen was due mainly to drug-related factors, improvement of health, financial buoyancy, the effectiveness of the drug, clarity of the information about the drugs and lots more than was mentioned by the respondents. Vermeire, Hearnshaw [22] also identified the absence of symptoms as a major hindrance to compliance. Most patients always feel that the temporary absence of symptoms indicate that they have been completely relieved from the condition. Hence, there is no need for taking the drug or complying with other therapeutics conditions. Failure to identify the chronic nature of the illness often serves as a barrier to adherence. Some patients viewed hypertension as an intermittent and recurring problem [30]. The failure to regard hypertension as a chronic problem requiring constant management imposes challenges with adherence to antihypertensive drugs. This finding remains consistent with findings from Kones [8] who asserted that two of the most important factors contributing to poor adherence were undoubtedly the asymptomatic and lifelong nature of the disease.

This may, however, be far from the truth. Especially for an illness like hypertension, the symptom may subside for a period which may be as long as ten years in which an individual may not manifest any symptom without taking any prescribed medication. Even though the individual seems to be free from illness symptom during this period, biomedical parameters may suggest that the illness is not completely cured. This is also corroborated by Kones [8] who opined that the patient's understanding and perception of hypertension is pertinent to compliance with treatment regimen. It has also been documented that patients' perceptions of the cause and course of hypertension may affect their ability to comply with treatment [30].

Dissatisfaction with service is responsible for poor compliance as identified by participants in this study. This may be a major challenge especially when care received falls below the expectation of patients. This is also supported by Mosadeghrad [31] who observed a similar finding in their study. Evidence exists for a correlation between education and physical health [32]. Even for the educated one, who may not be literate regarding treatment modalities, they found it difficult complying with treatment instruction. The situation may be worse when the patient has multi-morbidity and has to deal with all these diseases at the same time.

Our study asserts that affordability of services affect compliance. This supports Gulliford, Figueroa-Munoz [33] who opined that access to health services is closely linked to some certain factors including affordability, physical accessibility, and acceptability of services. The author further argued that this is not based merely on the adequacy of service supply but that the service must be relevant and effective if the population is to gain access to satisfactory health outcomes. According to Sienkiewicz [34] access to health services encompasses two things namely: health prevention and promotion. In addition, people must be empowered to demand appropriate health resources to protect or improve their health [34]. Social, financial and cultural barriers limit the availability and affordability of health services for such individuals or groups.

Notably, accurate information about treatment, enlightenment, technology and scientific orientation about hypertension is highly imperative for a positive outcome of the disease management. Patients with hypertension are often asymptomatic [35]. Siegel [35] further documented that the disease lacks a tangible reinforcement factor in fostering medication adherence in contrast with what may be seen in other chronic conditions such as diabetes where symptoms of hyperglycemia remind the patient about the need to take medication. Good relationships between the patients and their healthcare providers are fundamental for good adherence [29]. Empathetic and nonjudgmental attitude and assistance, readily available, good quality of communication and interaction have been shown to promote adherence to healthcare among adolescents [36].

Health systems-related issues affect the promotion of adherence among hypertensive patients [37]. These include limited and unavailable medications and out-of-pocket health care expenditure [37]. In addition, financing, affordable prices and reliable supply systems have been shown to have a direct influence on patient adherence, particularly in poorer segments of the population [38].

The complexity of treatment and healthcare setting is another factor associated with poor adherence. Kones [8] reported that frequency of dosing, number of concurrent medications and changes in anti-hypertensive medications as some health system-related factors. Finance has been a serious challenge in all facets of human endeavours and access to health care facilities with the absence of financial 
resources is inevitable. Worse still is the lack of health insurance for citizens who have to pay out-of-pocket. The situation may even be worse in the midst of dwindling economic situation in the country.

The results of this study should be considered in light of the main limitation of the study which is related to its small sample size. This is not unusual for a qualitative study. Therefore, our results may not be representative of all the hypertensive patients. However, findings from this study provide significant insights into the interplay of the day-today challenges of hypertensive patients in Nigeria and other countries with similar settings.

The findings of this study provide an objective data for assessing compliance with treatment among patients with hypertension. A holistic approach is needed to assess an individual's total experience of living with hypertension as opposed to focusing solely on physical care. The health worker should be vast in communicating effectively with the patient in other to get feedback which improves their level of drug and clinic compliance. Improvement is also expected in health education of patient to impart knowledge and improve change of behaviour in the issue relating to lifestyle modality and treatment modality

\section{Conclusion}

Compliance with treatment among hypertensive patients is essential to the positive outcome of the management of hypertension. This study concluded that lack of understanding of the chronic nature of hypertension hinders the participants from complying with treatment. It also identified inaccessible and poor quality services as a barrier to seeking treatment while the desire to regain functionality serves as a significant impetus for continuing with the treatment. Therefore, healthcare professionals should provide appropriate and informing health education to clients attending the clinic. There should also be a government intervention to subsidise drug and healthcare cost to make health services accessible and affordable for the patient. In addition, health insurance should be made functional and to accommodate the entire populace while drug should be put in simple and easy to read packages

\section{Conflict of Interest}

The authors declare that they have no competing interests.

\section{References}

[1] Gupta, R. and D. Xavier, Hypertension: The Most Important Non Communicable Disease Risk Factor in India. Indian Heart Journal, 2018.

[2] Akinroye, K., Nigerians wake up to high blood pressure. Bull World Health Organ, 2013. 91: p. 242-243.

[3] Anchala, R., et al., Hypertension in India: a systematic review and meta-analysis of prevalence, awareness, and control of hypertension. Journal of hypertension, 2014. 32(6): p. 1170.

[4] Akinlua, J. T., et al., Current prevalence pattern of hypertension in Nigeria: A systematic review. PloS one, 2015. 10(10): p. e0140021.

[5] Ajayi, I., et al., Blood pressure pattern and hypertension related risk factors in an urban community in Southwest Nigeria: The Mokola hypertension initiative project, Ibadan, Nigeria. Journal of Public Health and Epidemiology, 2017. 9(4): p. 51-64.

[6] Steel, Z., et al., The global prevalence of common mental disorders: a systematic review and meta-analysis 1980-2013. International journal of epidemiology, 2014: p. dyu038.

[7] Ogundipe, S. and C. Obinna, Hypertension: Why FG must act now, in Vanguard Newspaper, April 9 2013. 2013, Vanguard Newspaper: Lagos.

[8] Kones, R., Primary prevention of coronary heart disease: integration of new data, evolving views, revised goals, and role of rosuvastatin in management. A comprehensive survey. Drug design, development and therapy, 2011. 5: p. 325.

[9] Martin, L. R., et al., The challenge of patient adherence. Ther Clin Risk Manag, 2005. 1(3): p. 189-199.

[10] Vrijens, B., et al., Current situation of medication adherence in hypertension. Frontiers in pharmacology, 2017. 8: p. 100.

[11] van Boven, J. F., et al., Clinical and economic impact of nonadherence in COPD: a systematic review. Respiratory medicine, 2014. 108(1): p. 103-113.

[12] Jin, J., et al., Factors affecting therapeutic compliance: A review from the patient's perspective. Therapeutics and clinical risk management, 2008. 4(1): p. 269.

[13] Martin, L. R., et al., The challenge of patient adherence. Therapeutics and clinical risk management, 2005. 1(3): p. 189.

[14] Amira, C. and N. Okubadejo, Factors influencing noncompliance with anti-hypertensive drug therapy in Nigerians. The Nigerian postgraduate medical journal, 2007. 14(4): p. 325-329.

[15] Kabir, M., et al., Compliance to medication among hypertensive patients in Murtala Mohammed Specialist Hospital, Kano, Nigeria. Journal of Community medicine and primary health care, 2004. 16(1): p. 16-20.

[16] Osamor, P. E. and B. E. Owumi, Factors associated with treatment compliance in hypertension in southwest Nigeria. J Health Popul Nutr, 2011. 29(6): p. 619-28.

[17] Becker, M. H., The health belief model and sick role behavior. Health Education \& Behavior, 1974. 2(4): p. 409-419.

[18] Hsieh, H.-F. and S. E. Shannon, Three approaches to qualitative content analysis. Qualitative health research, 2005. 15(9): p. 1277-1288.

[19] Strauss, A. and J. Corbin, Basics of qualitative research: Procedures and techniques for developing grounded theory. 1998, Thousand Oaks, CA: Sage.

[20] Sahay, S., K. S. Reddy, and S. Dhayarkar, Optimizing adherence to antiretroviral therapy. Indian Journal of Medical Research, 2011. 134(6): p. 835. 
[21] World Health Organization Stop TB Initiative, Treatment of tuberculosis: guidelines. 2010: World Health Organization.

[22] Vermeire, E., et al., Patient adherence to treatment: three decades of research. A comprehensive review. Journal of clinical pharmacy and therapeutics, 2001. 26(5): p. 331-342.

[23] De Pascual-Verdú, R., M. Rocha-Díaz, and G. AlmazánAntón, Relevant factors in treatment adherence: A case study. Clínica y Salud, 2015. 26(3): p. 141-150.

[24] Brown, M. T. and J. K. Bussell. Medication adherence: WHO cares? In Mayo Clinic Proceedings. 2011. Elsevier.

[25] Haddad, P. M., C. Brain, and J. Scott, Nonadherence with antipsychotic medication in schizophrenia: challenges and management strategies. Patient Relat Outcome Meas, 2014. 5: p. $43-62$.

[26] DiMatteo, M. R., K. B. Haskard, and S. L. Williams, Health beliefs, disease severity, and patient adherence: a metaanalysis. Medical care, 2007. 45(6): p. 521-528.

[27] Akoko, B. M., et al., Knowledge of hypertension and compliance with therapy among hypertensive patients in the Bamenda Health District of Cameroon: a cross-sectional study. Cardiology and therapy, 2017. 6(1): p. 53-67.

[28] Jurkiewicz, M. T., S. Marzolini, and P. Oh, Adherence to a home-based exercise program for individuals after stroke. Topics in stroke rehabilitation, 2015.

[29] Sabaté, E., Adherence to long-term therapies: evidence for action. 2003: World Health Organization.

[30] Bokhour, B. G., et al., The role of patients' explanatory models and daily-lived experience in hypertension selfmanagement. Journal of general internal medicine, 2012. 27(12): p. 1626-1634.

[31] Mosadeghrad, A. M., Factors influencing healthcare service quality. International journal of health policy and management, 2014. 3(2): p. 77.

[32] Zimmerman, E. B., S. H. Woolf, and A. Haley, Understanding the relationship between education and health: a review of the evidence and an examination of community perspectives. Population health: behavioral and social science insights. Rockville (MD): Agency for Health-care Research and Quality, 2015: p. 347-84.

[33] Gulliford, M., et al., What does' access to health care'mean? Journal of health services research \& policy, 2002. 7(3): p. 186-188.

[34] Sienkiewicz, D., Access to Health Services in Europe. European Social Watch Report, 2010. 2010: p. 17-20.

[35] Siegel, D., Barriers to and strategies for effective blood pressure control. Vasc Health Risk Manag, 2005. 1(1): p. 9-14.

[36] Taddeo, D., M. Egedy, and J.-Y. Frappier, Adherence to treatment in adolescents. Paediatr Child Health, 2008. 13(1): p. 19-24.

[37] Malik, A., et al., Hypertension-related knowledge, practice and drug adherence among inpatients of a hospital in Samarkand, Uzbekistan. Nagoya journal of medical science, 2014. 76(3-4): p. 255.

[38] Gray, A., Access to medicines and drug regulation in developing countries: a resource guide for DFID. London: DFID Health Systems Resource Centre, 2004. 